\title{
Resistance of Clostridium perfringens to $\beta$-Lactam Antibiotics Mediated by a Decreased Affinity of a Single Essential Penicillin-binding Protein
}

\author{
By RUSSELL WILLIAMSON \\ Laboratoire de Microbiologie du Professeur Acar, Institut Biomédical des Cordeliers, \\ 75270 Paris, France
}

(Received 29 March 1983)

\begin{abstract}
Benzylpenicillin-resistant mutants of Clostridium perfringens have been isolated by in vitro selection. The sole mechanism of resistance was a decreased affinity of the highest molecular weight penicillin-binding protein (PBP1) for the antibiotic.
\end{abstract}

\section{INTRODUCTION}

Clostridium perfringens grows as filaments in the presence of low concentrations (below the MIC) of $\beta$-lactam antibiotics, and this is due to inhibition of septum formation (Gardner, 1940; Crofts \& Evans, 1950). The four lowest molecular weight penicillin-binding proteins (PBPs) of the six in this species (Murphy et al., 1981), became virtually saturated with benzylpenicillin when the organism was grown with a concentration of this antibiotic sufficient to cause filamentation of an entire population (Williamson \& Ward, 1982). This morphological effect occurred with no inhibition of the increase of cell mass, and there was no detectable binding of the antibiotic to the highest molecular weight PBPs. Thus, it was suggested that PBP1 and/or PBP2 could have the essential transpeptidase activity responsible for the continued incorporation of peptidoglycan and growth of the bacteria (Williamson \& Ward, 1982). Since there have been no reports of $\beta$-lactamase activity in $C$. perfringens, and the walls of several Gram-positive bacteria do not appear to be permeability barriers to $\beta$-lactam antibiotics (Gutmann et al., 1981; Williamson et al., 1983), it could be expected that mutants resistant to benzylpenicillin might have high molecular weight PBPs with reduced affinity for the antibiotic as the sole basis for resistance.

\section{METHODS}

Clostridium perfringens strain 667 was obtained from Dr Madeleine Sebald, Institut Pasteur, Paris, and grown as previously described (Williamson \& Ward, 1982). Spontaneous, benzylpenicillin-resistant mutants were selected initially on plates (Brain Heart Infusion, Difco) containing the MIC of this antibiotic for the parental strain. Thereafter, the mutants were selected by stepwise adaptation to twofold increasing concentrations of benzylpenicillin in liquid medium, using 1-1000 dilutions of exponential-phase bacteria from the previous selection step. At each resistance level, the strains were isolated, purified, and the resistance level to benzylpenicillin verified before selection of the next mutant in the series. In addition, only strains that grew with normal morphology (rods) in the absence of antibiotic were used in the selection procedure.

MICs of $\beta$-lactam antibiotics were determined using a twofold agar dilution technique, with a replicating device (Steers et al., 1959) and approximately $10^{3}$ and $10^{4}$ exponential-phase bacteria per spot. The antibiotics were obtained as follows: benzylpenicillin (Pen G), Specia, Paris; carbenicillin (Carb), Beecham-Sevigne, Paris; cephalothin (Thin) and ceftazidime (Taz), Glaxo; moxalactam (Mox), Eli Lilly, S.A., St Cloud, France; oxacillin (Oxa), Bristol Laboratories, Paris; cefoperazone (Peraz), Pfizer; ceftriaxone (Trx), Roche, S.A., Neuilly, France; and cefotaxime (Tax), from Roussel-Uclaf, Romainville, France. Nitrocefin was the generous gift of Dr Hotston, Glaxo Research, Greenford, Middlesex. $p$ - $\left[{ }^{3} \mathrm{H}\right]$ Benzylpenicillin $\left(26 \mathrm{Ci} \mathrm{mmol}^{-1}, 0.66 \mathrm{TBq} \mathrm{mmol}^{-1}\right)$ was obtained

Abbreviations: MIC, minimum inhibitory growth concentration; PBP, penicillin-binding protein. 
from Rhône-Poulenc C.N.G., Vitry, France. Discs containing $30 \mu \mathrm{g}$ of chloramphenicol, rifampicin, and vancomycin were purchased from BioMérieux, Paris.

PBPs were detected in whole bacteria as previously described (Williamson \& Ward, 1982). The band densities of the fluorograms were quantified with a Helena Laboratories Cliniscan (Beaumont, Tex., U.S.A.).

\section{RESULTS}

A series of ten increasingly benzylpenicillin-resistant strains were obtained as spontaneous mutants from wild-type bacteria, each mutant being derived from the preceding less-resistant strain. The mutants also became progressively more resistant to all the $\beta$-lactam antibiotics tested (Table 1). The increase in MIC was least $(16 \times)$ for carbenicillin and greatest (at least $8500 \times$ ) for the third-generation cephalosporins, ceftriaxone, cefotaxime, and ceftazidime. In contrast, each strain retained wild-type sensitivity to chloramphenicol, rifampicin, and vancomycin (data not shown). No $\beta$-lactamase activity was detected in any strain, whether or not the bacteria were grown with any of the $\beta$-lactam antibiotics tested in Table 1 or with cefoxitin (data not shown). It was found that as the resistance to benzylpenicillin increased, the growth rate of cultures without any antibiotic decreased significantly in comparison with that of the parental strain (Table 1). The most resistant strain $(P 2 \cdot 0)$ grew at only about half the rate of the wild-type strain (667).

All of the strains grew as filaments in medium containing benzylpenicillin at half the respective MIC of this antibiotic for each mutant, and also at half the MIC for the wild-type, parental strain (data not shown). Similar results were obtained with all of the other $\beta$-lactam antibiotics. The effect of benzylpenicillin was reversed in all cases by subsequent addition of penicillinase.

Examination of the PBPs revealed that the apparent affinity of only PBP1 decreased as the resistance of the strains increased (Fig. 1). None of the other five PBPs (2-6) appeared changed in any of the mutants. In fact, both the relative amounts and the affinities of these PBPs were virtually identical in each strain, indicating that no changes had occurred in these proteins. Calculation of the total amount of PBP1 in each strain under saturating $\left[{ }^{3} \mathrm{H}\right]$ benzylpenicillin conditions showed no significant differences, but the concentrations $\left(\mu \mathrm{g} \mathrm{ml}^{-1}\right)$ of antibiotic required to produce $50 \%$ saturation of this protein in the wild-type and mutant strains were: wild-type (667), $0.90 ; P 0 \cdot 03,0.85 ; P 0 \cdot 06,0.95, P 0 \cdot 125,1 \cdot 1 ; P 0 \cdot 25,1.35 ; P 0 \cdot 5,3 \cdot 2 ; P 0 \cdot 75,5 \cdot 8$; $P 1 \cdot 0,6 \cdot 2 ; P 1 \cdot 25,6 \cdot 8 ; P 1 \cdot 5,7 \cdot 4$; and $P 2 \cdot 0,7 \cdot 6$.

Table 1. Antibiotic sensitivities of wild-type and benzylpenicillin-resistant mutants of C. perfringens

Antibiotic MIC $\left(\mu \mathrm{g} \mathrm{ml}^{-1}\right)$

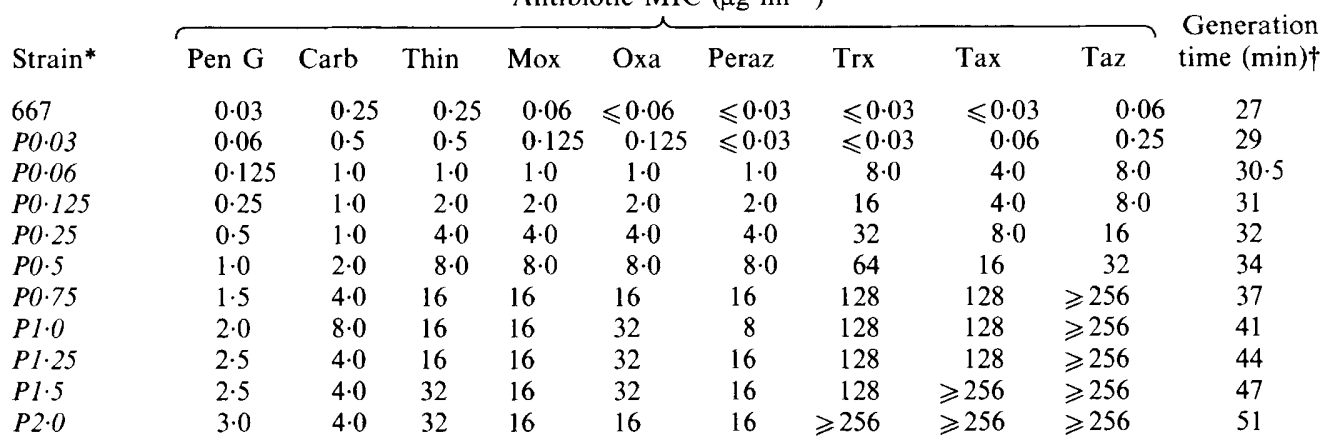

* The strain designation indicates the highest concentration of benzylpenicillin in which the mutant could grow. † Generation times were measured in liquid medium without antibiotics. 
Antibiotic

concn $\left(\mu \mathrm{g} \mathrm{ml}^{-1}\right)$

30

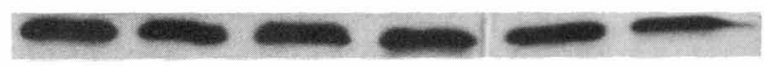

10

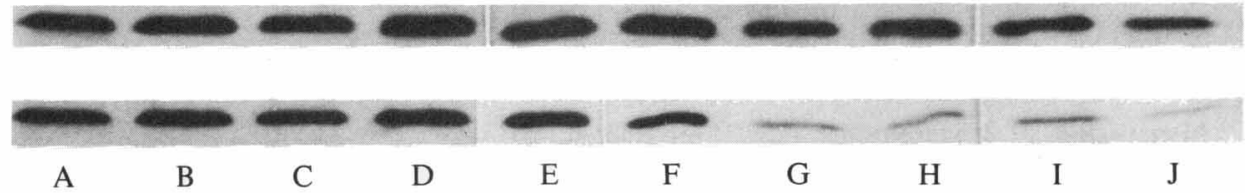

Fig. 1. Binding of $\left[{ }^{3} \mathrm{H}\right]$ benzylpenicillin to PBPI from wild-type and resistant mutants of $C$, perfringens. PBPs were detected in whole bacteria using the concentrations of antibiotic indicated, and the bands of PBPl from the fluorograms were cut out for the figure. A, wild-type (667); B, P0.03; C, P0.06; D, $P 0 \cdot 125 ; \mathrm{E}, P 0 \cdot 25 ; \mathrm{F}, P 0 \cdot 5 ; \mathrm{G}, P 0 \cdot 75 ; \mathrm{H}, P 1 \cdot 0 ; \mathrm{I}, P l \cdot 5 ; \mathrm{J}, P 2 \cdot 0$.

\section{DISCUSSION}

The resistance of Gram-positive bacteria to $\beta$-lactam antibiotics by an apparent reduction in affinity of one or more PBPs has been reported for Staphylococcus aureus (Brown \& Reynolds, 1980; Hayes et al., 1981), Streptococcus pneumoniae (Zighelboim \& Tomasz, 1980), Bacillus subtilis (Buchanan \& Strominger, 1976), and Bacillus megaterium (Giles \& Reynolds, 1979). High molecular weight PBPs were involved in the resistance of $B$. subtilis and B. megaterium, and thus PBP1 of $C$. perfringens appears to be a similar target and may therefore be the $\beta$-lactam antibiotic-sensitive primary transpeptidase. However, in $C$. perfringens the affinity of only one PBP was affected, whereas the other bacilli had additional changes in the apparently nonessential PBPs (Buchanan \& Strominger, 1976; Giles \& Reynolds, 1979). Since the total amount of PBP1 in C. perfringens was essentially the same in each strain, the decreasing growth rate as the resistance increased may indicate that the activity of this protein may become rate limiting for growth of the bacteria. It is possible that the affinity of PBP1 for the natural substrate in the transpeptidase reaction would be reduced in proportion to the decreased affinity for benzylpenicillin.

The significantly decreased activities of the third-generation cephalosporins in comparison with previously introduced $\beta$-lactam antibiotics for the resistant mutants in the present study may suggest that their structure does not allow a sufficiently good interaction with the active site of the presumed transpeptidase (PBP1). This difference was particularly evident in the mutants resistant to 0.03 and $0.06 \mu \mathrm{g}$ benzylpenicillin $\mathrm{ml}^{-1}$, which were distinguished by some 32- to 266fold decrease in their susceptibility to ceftriaxone, cefotaxime, or ceftazidime.

The lack of additional changes in the lower molecular weight PBPs (2-6) of the mutants in $C$. perfringens, adds further evidence that these proteins are not of primary importance to the organism (Williamson \& Ward, 1982). Since each of the strains grew as filaments in the same concentrations of $\beta$-lactam antibiotics as the parental strain, as well as at half the respective MIC of each antibiotic for each mutant, it is likely that the function(s) of these PBPs were the same in all strains, and that the inhibition of septation was due to inhibition of one or more of these proteins. This filamentous response does not appear to be lethal for the organism because the bacteria grew at the same rate, and also formed normal-sized colonies, at sub-MICs of all $\beta$ lactam antibiotics. This is in contrast with Escherichia coli, in which the inhibition of PBP3 by a relatively PBP-specific antibiotic caused filamentation, and this was apparently lethal (Shah et al., 1981; Georgopapadakou et al., 1982). However, Chase et al. (1981) have shown that saturation of PBP3 alone may not be sufficient for cell death in E. coli.

The morphological response of $C$. perfringens at low antibiotic concentrations and the same interactions of $\left[{ }^{3} \mathrm{H}\right]$ benzylpenicillin with the lower molecular weight PBPs (2-6) in each strain, indicates that there was no significant permeability barrier to the antibiotic. Thus, the resistance 
was apparently solely due to the decrease in affinity of the PBP1, which is likely to be the primary transpeptidase.

I thank Drs J. Barrie Ward and Laurent Gutmann for helpful discussions. This work was supported by grants from INSERM and l'Office Technique du Territoire.

\section{REFERENCES}

Buchanan, C. E. \& Strominger, J. L. (1976). Altered penicillin-binding components in penicillin-resistant mutants of Bacillus subtilis. Proceedings of the National Academy of Sciences of the United States of America 73, 1816-1820.

Brown, D. F. J. \& ReYNolds, P. E. (1980). Intrinsic resistance to $\beta$-lactam antibiotics in Staphylococcus aureus. FEBS Letters 122, 275-278.

Chase, H. A., Fuller, C. \& Reynolds, P. E. (1981) The role of penicillin-binding proteins in the action of cephalosporins against Escherichia coli and Salmonella typhimurium. European Journal of Biochemistry 117, 301-310.

Crofts, J. E. \& Evans, D. G. (1950). The action of penicillin on Clostridium welchii type A. British Journal of Experimental Pathology 31, 550-561.

GARDNER, A. D. (1940). Morphological effects of penicillin on bacteria. Nature, London 146, 837-838.

Georgopapadakou, N. H., Smith, S. A. \& Sykes, R. B. (1982). Mode of action of azthreonam. Antimicrobial Agents and Chemotherapy 21, 950-956.

Giles, F. A. \& ReYnolds, P. E. (1979). Bacillus megaterium resistance to cloxacillin accompanied by a compensatory change in penicillin-binding proteins. Nature, London 280, 167-168.

Gutmann, L., Williamson, R. \& Tomasz, A. (1981). Physiological properties of penicillin-binding proteins in group A streptococci. Antimicrobial Agents and Chemotherapy 19, 872-880.
Hayes, M. V., Curtis, N. A. C., Wyke, A. W. \& WARD, J. B. (1981). Decreased affinity of a penicillin-binding protein for $\beta$-lactam antibiotics in a clinical isolate of Staphylococcus aureus resistant to methicillin. FEMS Microbiology Letters 10, 119-122.

Murphy, T. F., Barza, M. \& Park, J. T. (1981). Penicillin-binding proteins in Clostridium perfringens. Antimicrobial Agents and Chemotherapy 20, 809-813.

Shah, P. M., Losert-Bruggner, B. \& Stille, W. (1981). Bactericidal activity of SQ 26,776. Journal of Antimicrobial Chemotherapy 8 (Suppl. E), 77-80.

Steers, E., Foltz, E. L. \& Graves, B. S. (1959). An inocula replicating apparatus for routine testing of bacterial susceptibility to antibiotics. Antibiotics and Chemotherapy 9, 307-311.

WILliamson, R. \& WARD, J. B. (1982). Benzylpenicillin-induced filament formation of Clostridium perfringens. Journal of General Microbiology 128, 30253035.

Williamson, R., Calderwood, S. B., Moellering, R. C., JR. \& Tomasz, A. (1983). Studies on the mechanism of intrinsic resistance to $\beta$-lactam antibiotics in group D streptococci. Journal of General Microbiology 129, 813-822.

ZighelboIM, S. \& TomasZ, A. (1980). Penicillinbinding proteins of multiply antibiotic-resistant South African strains of Streptococcus pneumoniae. Antimicrobial Agents and Chemotherapy 17, 434-442. 